\title{
Um estudo sobre a transição do ensino das noções de funções trigonométricas entre o ensino médio e superior no Brasil e França
}

A study of the transition of trigonometric functions concepts from secondary to higher education in Brazil and France

\author{
L. S. Fonseca ${ }^{1 *}$; L. G. X. Barros ${ }^{2}$ \\ ${ }^{1}$ Coordenadoria de Licenciatura em Matemática, IFS, 49055-260, Aracaju-Sergipe, Brasil \\ ${ }^{1}$ Programa de Pós-Graduação em Educação Matemática, UNIAN-SP, 02022-011, São Paulo-São Paulo, Brasil
}

*laerte.fonseca@uol.com.br

(Recebido em 08 de março de 2016; aceito em 08 de junho de 2016)

\begin{abstract}
O presente artigo é resultado de uma pesquisa de tese de doutorado que procurou analisar a transição do ensino das noções de funções trigonométricas entre o Ensino Médio e o Ensino Superior, sob a ótica da articulação entre quadros da Didática da Matemática e da Neurociência Cognitiva. Os referenciais teóricos adotados foram os fundamentos da Teoria Antropológica do Didático de Chevallard e da abordagem dos Níveis de Funcionamento do Conhecimento de Robert para o entendimento da Atividade Matemática no meio institucional e, no meio cerebral, considerando as perspectivas teóricas da Neurociência Cognitiva de Kandel. Realizados os exames nos níveis de Ensino Médio e Superior por meio de documentos oficiais do ensino brasileiro e francês, em livros didáticos de matemática de ambas as nacionalidades e do processamento cerebral da Atividade Matemática por meio da Memória de Longo Prazo (MLP) em estudantes do Brasil e da França, é possível afirmar que a transição em tela nos dois países ocorre de maneira lenta e média, respectivamente, considerando os documentos oficiais; na mesma sequência, de modo médio e alto, analisando-se os livros didáticos e, por fim, em ritmo médio nos dois territórios, quando enfocou-se nos ostensivos e não ostensivos vinculados as noções de funções trigonométricas existentes e disponíveis na MLP dos estudantes investigados. Esse conjunto de fatos permitiu concluir que a transição do ensino das noções de funções trigonométricas apesar de acontecer nos países analisados de modos diferentes, preserva uma causa singular. Além disso, não consideram institucionalizados os princípios da Neurociência Cognitiva como uma possibilidade para ajudar a neutralizar o tipo de ruptura existente.

Palavras-chave: Transição; Ensino Médio e Superior, Funções Trigonométricas
\end{abstract}

In this article are presented results of a doctorate thesis research aiming to analyze of the transition of trigonometric functions concepts from secondary to higher education from the perspective of the relationship between cadres of the didactics of mathematics and Cognitive Neuroscience. The theoretical references adopted were the fundamentals of Anthropological Theory of Didactic of Chevallard and the approach of Robert Knowledge Functioning Levels for the understanding of mathematics activity in the institutional environment and in the brain, considering the theoretical perspectives of Cognitive Neuroscience of Kandel. Conducted the tests in levels of high school and higher through official documents of Brazilian education and French in textbooks of mathematics of both nationalities and the brain processing of Mathematics Activity through the Long Term Memory (LTM) for students Brazil and France, it is clear that the transition of the two countries takes place slowly and medium, respectively, considering the official documents; in the same sequence, medium and high mode, textbooks are analyzing and, finally, on average pace in both territories, when focused to the ostensible and not ostentatious linked the notions of existing trigonometric functions and available in MLP students investigated. This set of facts showed that the transition of teaching trigonometric functions notions despite happen in the analyzed countries in different ways, preserves a singular cause. Also, they do not consider institutionalized the principles of Cognitive Neuroscience as a possibility to help neutralize the existing type of rupture.

Keywords: Transition, High school and Higher education, Trigonometric functions 


\section{INTRODUÇÃO}

As pesquisas de transição entre os níveis de ensino de matemática têm possibilitado importantes resultados que possibilitam propor alguns direcionamentos metodológicos à referida área do conhecimento.

Nesta perspectiva, o artigo em tela buscou desvelar um estudo específico que analisou a transição do ensino das noções de funções trigonométricas entre o Ensino Médio e o Ensino Superior sob a ótica da articulação entre quadros da Didática da Matemática e da Neurociência Cognitiva.

Como o efeito da abordagem de tais noções está atrelado à existência de um tratamento didático, evocaram-se as concepções de Chevallard [1,2] e de Robert [3, 4] para analisar, a organização matemática existente nos documentos oficiais dos sistemas de ensino brasileiro e francês, bem como seus níveis de funcionamento do conhecimento em estudantes do Ensino Superior, cuja Atividade Matemática estava disponível na disciplina Cálculo I.

Essa disponibilidade também foi avaliada pela existência e relação de objetos ostensivos e não ostensivos identificados por meio da ativação da Memória de Longo Prazo (MLP), considerando as perspectivas teóricas da Neurociência Cognitiva de Kandel [5].

Desta forma, estruturou-se o presente texto, privilegiando reflexões acerca do ensino das noções em jogo, dos quadros teóricos relacionados e destacando-se os principais resultados obtidos na investigação de Fonseca [6].

Em síntese, verificou-se que a transição do ensino das noções de funções trigonométricas apresenta-se de modos diferentes entre as nacionalidades analisadas, embora as rupturas encontradas partam do mesmo gatilho: mudança de técnicas $(\tau)$ empregadas entre a evolução dos estágios epistemológicos das referidas noções. Principalmente, por essas técnicas $(\tau)$ não serem ativadas sob um mesmo discurso tecnológico $(\theta)$, quando eram articuladas as noções de ângulos e a noção de número real. E, por outro lado, quando os tipos de Tarefas associados às atividades matemáticas relacionadas as noções em jogo resistirem considerar a institucionalização dos princípios da Neurociência Cognitiva como uma possibilidade para ajudar a neutralizar o tipo de ruptura existente.

\subsection{Sobre o ensino das noções de funções trigonométricas}

Como um tema matemático, as noções de funções trigonométricas resultam de um processo amalgamado pelos domínios da geometria e da álgebra. Da geometria porque permitiu a identificação de relações entre lados e ângulos de um triângulo, fundando, dessa forma, o campo da trigonometria (KENNEDY, 1992) [7]. Do lado da álgebra, por estabelecer relações funcionais entre grandezas vinculadas aos fenômenos ondulatórios, especificamente, as oscilações mecânicas (SAMPAIO e CALÇADA, 2008) [8].

Nestes termos, o presente tema matemático desenvolveu-se naturalmente sob o abrigo epistemológico de sua época, expandindo-se até o início do século $\mathrm{XX}$, quando os físicos sentiram a necessidade de aprimorar os métodos mecânicos para compreensão do som. Dentre esses dispositivos, exemplifica-se, conforme Abdounur [9], o Phonodeik - pode ser descrito como sendo um "dispositivo mecânico que registra fotograficamente formas sonoras, [...], possui relevante importância na comprovação da natureza periódica do som", - inventado por Dayton C. Miller em 1908. Naquela época, o Teorema de Fourier ou Teorema da Soma de funções trigonométricas pôde ser melhor compreendido como resultado de um processo de Modelagem Matemática mais aproximada da realidade ora investigada.

Com efeito, essa breve digressão ao passado se justifica por considerar importante ao ensino das noções em tela as orientações disponíveis nos Parâmetros Curriculares Nacionais do Ensino Médio (PCNEM) que objetiva o desenvolvimento de conhecimentos práticos, contextualizados, que respondam às necessidades da vida contemporânea, e o desenvolvimento de conhecimentos mais amplos e abstratos, que correspondam a uma cultura geral e a uma visão de mundo (BRASIL, 2000) [10].

No estudo de Fonseca [6], foi considerado como um obstáculo à aprendizagem do referido tema a "sobrevivência" e "funcionamento" das organizações praxelógicas, quando identificadas, 
nos documentos oficiais (programas de ensino e livros didáticos) existentes e disponíveis no sistema educativo do Brasil e da França. Para Nasser, Sousa e Torraca [11], esse tipo específico de obstáculo contribui para elevar os índices de evasão e repetência na disciplina Cálculo I do Ensino Superior e, geralmente, decorrem de lacunas na aprendizagem matemática da Educação Básica.

Em favor dessa reflexão, Rezende [12] reforça a necessidade de proteger o recuo à natureza epistemológica recomendada nas expectativas institucionais dos PCNEM para tentar contornar as possíveis dificuldades de aprendizagem em Cálculo I, e sugere que as tarefas desenvolvidas no Ensino Médio sejam elaboradas em termos de problemas sobre variabilidade de funções.

Como sugestão metodológica desse contexto, Palis [13] aponta o trabalho com softwares livres, como uma ferramenta para ajudar na apropriação da articulação entre as representações "algébricas e gráficas" das funções trigonométricas. Essa opção de ensino pode, conforme Sierpinska [14], auxiliar na diminuição dos entraves decorrentes da falta de conhecimentos prévios dos estudantes, que contribuiriam na identificação das conexões entre geometria e álgebra esperadas para a compreensão das noções aqui discutidas.

Ainda em Fonseca [6], outro ponto de vista é lançado sobre a problemática do ensino das noções das funções trigonométricas: as dificuldades de aprendizagem em Cálculo I podem decorrer, também, da qualidade da evocação de noções e manipulação de propriedades relativas às funções trigonométricas necessárias para resolverem as indeterminações dos limites trigonométricos $[15,16]$. Essa constatação procedeu dos resultados de pesquisas anteriores do mesmo autor, que mobilizou interesse para analisar a qualidade das referidas informações por meio da compreensão do processamento cerebral, especificamente, pelo funcionamento da Memória de Longo Prazo.

Desse modo, foi possível justificar a contemporaneidade e inclusão das perspectivas da Neurociência Cognitiva - como uma área vanguardista de pesquisas sobre a aprendizagem - para questionar as variações ou níveis de aprendizagem das noções de funções trigonométricas disponíveis nos documentos oficiais, segundo a possibilidade dos moldes da Teoria Antropológica do Didático (TAD), ou seja, de uma organização praxeológica que "exista", "funcione" e "sobreviva" em uma determinada ecologia de tarefas presentes no sistema de ensino brasileiro e francês.

No próximo tópico, será abordado sobre as duas vertentes teóricas a fim de auxiliar o leitor sobre o uso de ambas em cenários de ensino ou de aprendizagem matemática, especificamente, quando se analisou a transição Ensino Médio-Ensino Superior relativa ao ensino das noções de funções trigonométricas em territórios diferenciados pela própria cultura.

Sob o abrigo das reflexões acima, foi possível identificar, no estudo de Fonseca [6], algumas fontes das etiologias das dificuldades de aprendizagem quando as lentes do pesquisador são aplicadas sobre o ensino das noções de funções trigonométricas.

O objetivo deste levantamento foi compreender como e a partir de que as dificuldades de aprendizagem (matemática) são analisadas. As fontes representam trabalhos de pesquisa (Dissertações e Teses) pinçados de Programas de Pós-Graduação (PPG) sob o critério de investigação da temática dificuldade de aprendizagem escolar (DA/DAE) e/ou dificuldade de aprendizagem matemática/funções trigonométricas (DAMFT). Dentre esses trabalhos, encontraram-se alguns resultados no intervalo cronológico 1987 - 2011: Educação (E), Psicologia (P), Neurociência (NC) e Educação Matemática (EM).

Em síntese, foi possível constatar sinais (palavras ou expressões negritadas) de Neurociência em todos os marcadores e, consequentemente, em todos os programas de Pós-Graduação pesquisados. Dessa forma, reforça-se a justificativa de que existe a possibilidade e necessidade de analisar a Atividade Matemática considerando tanto o meio externo (as instituições) como o meio interno (o cérebro).

Considerando os resultados sobre as causas das dificuldades de aprendizagem das noções de funções trigonométricas e suas possíveis implicações para transição Ensino Médio-Ensino Superior (EM-ES), supõe-se que a propagação de tal fenômeno, prejudicial à transição EM-ES, está vinculada à existência de uma ruptura dessa transição, bem como seu ensino, tanto no Ensino Médio como no Ensino Superior, não considera os princípios da Neurociência Cognitiva para 
diminuir tais dificuldades. Fundamentando-se nestas reflexões, emergiram as seguintes questões de pesquisa:

Q1 - Quais são as possíveis causas dessa ruptura?

Q2 - A institucionalização de atividades matemáticas baseadas nos princípios da Neurociência Cognitiva pode auxiliar no processo de neutralização dessa ruptura?

Tomando essas questões como fio condutor, foi preciso que a investigação considerasse a seguinte metodologia: breve apresentação dos quadros teóricos escolhidos: meio externo (institucional) - Teoria Antropológica do Didático (TAD) de Yves Chevallard, abordagem dos Níveis de Funcionamento do Conhecimento (NFC) de Aline Robert [3,4], que serviram como articuladores para compreender o meio interno (cérebro) - perspectivas teóricas da Neurociência Cognitiva, sob as lentes de Eric Kandel [5], principalmente; análise epistemológica das noções em jogo; as análises institucionais no Brasil e na França; análise neurocognitiva.

No presente artigo será apresentado o arranjo teórico entre os quadros anunciados e alguns resultados desses procedimentos entendendo serem suficientes para caracterizar os principais aspectos do processo da transição em jogo.

\subsection{Quadros teóricos relacionados na pesquisa: Teoria Antropológica do Didático, abordagem dos níveis de funcionamento do conhecimento e perspectivas teóricas da neurociência cognitiva}

Dentre as opções teóricas, disponíveis para fundamentar as análises sobre a transição do Ensino Médio para o Ensino Superior (EM-ES), enfocando os conhecimentos desenvolvidos no Ensino Médio sobre as Funções Trigonométricas (conhecimentos prévios) como possíveis suportes para a introdução de novos conhecimentos no Ensino Superior, centrou-se, na primeira etapa da investigação sobre os fundamentos da Teoria Antropológica do Didático - TAD por entender-se que reúne elementos possivelmente capazes de radiografar e tornar visíveis as relações latentes no cenário da Atividade Matemática (AM) que, nesse olhar, podem justificar a instabilidade das perspectivas institucionais e pessoais quando se reflete sobre o fracasso escolar em Matemática.

Sendo a TAD, uma teoria pertencente ao campo de pesquisa da Didática da Matemática, considera as noções de relações institucionais e pessoais, a noção de praxeologia, a noção de ostensivos e não ostensivos, ecologia e os níveis de co-determinação, segundo definições de Chevallard $(17,1,2,18,19,20)$ e Bosch e Chevallard [21] definem como necessários para analisar a AM em quaisquer circunstâncias.

Neste sentido, cabe ainda salientar que a TAD viabilizará, com precisão, o reconhecimento das praxeologias existentes nos documentos oficiais e também as expectativas institucionais, as expectativas tanto dos professores do Ensino Médio como do Superior acerca dos conhecimentos anteriores dos estudantes referentes à noção de Funções Trigonométricas, na passagem entre essas duas etapas escolares.

Por esses motivos e, a partir das noções de ostensivos e não ostensivos observou-se à necessidade de agregar uma abordagem auxiliar a TAD, teoria central da pesquisa, por entenderse que juntas possibilitarão mais consistência acerca do objeto em análise. Destaca-se, então, a noção dos três Níveis de Funcionamento do Conhecimento (NFC) dos estudantes idealizados por Robert [3, 4].

Esse enfoque concebido por Robert [3, 4], ilumina o cenário das articulações entre os NFC, relativos à noção de Funções Trigonométricas e, além disso, dá possibilidade para compreensão das mudanças ocorridas por meio das exigências institucionais, respectivas à transição das fases escolares anteriores.

Neste contexto, é esperado pelos estudantes do referido nível escolar que, através da flexibilidade cognitiva dos conteúdos matemáticos, possam ampliar, com autonomia, diversos tipos de tarefas (escolares ou profissionais) sugeridas e que determinem ocasionalmente a utilização de noções matemáticas, em particular, as de Funções Trigonométricas.

Essa mesma pesquisadora defende que tal autonomia vincula-se diretamente, de uma flexibilidade cognitiva que, dentro da área de Matemática, foi associada à capacidade de manipular uma variedade de ostensivos e evocar os não ostensivos associados. 
Dessa forma, postula-se que para dispor livremente dessa autonomia, é esperado dos estudantes, conforme Robert [3, 4], o NFC classificado como disponível, já que se faz necessário os diversos contextos matemáticos envolvidos numa tarefa dada.

A segunda etapa repousa sobre as perspectivas teóricas da Neurociência Cognitiva, introduzida nessa pesquisa por investigar a natureza do fenômeno da cognição e da aprendizagem. Ambos os fenômenos assessoram e sediam a AM processada no cérebro (meio interno) do aluno, importando compreender como esse conhecimento está ou não sendo considerado pelas teorias da Didática da Matemática, TAD e NFC, sobretudo, por essa última, vincular aos seus postulados a denominação flexibilidade cognitiva, terminologia específica da Neurociência Cognitiva.

Apesar de sua longa história, a área da Neurociência Cognitiva obteve essa denominação no final da década de 1970, conforme seu criador Gazzaniga [22], a partir dos estudos direcionados às funções cerebrais mais complexas como a cognição, linguagem, memória e aprendizagem.

As investigações buscavam analisar onde e como tais funções cerebrais ou cognitivas se estabeleciam no encéfalo, para que o comportamento humano fosse, cada vez melhor explicado. Para tanto, investimentos em equipamentos para explorar o cérebro permitiu o desenvolvimento da análise do tipo molecular para explicar como essas funções cognitivas se comunicam, processando a informação proveniente do meio externo.

Fundamentando-se em Kandel [5], sabe-se que células especiais denominadas de neurônios são responsáveis pela síntese de proteínas e por meio dos seus mensageiros, os neurotransmissores, processam a informação nas sinapses neuronais. Sendo a informação classificada como "intencional", sobretudo, as escolarizadas e direcionadas para a AM, interessa analisar se os NFC diluídos nas tarefas propostas nos documentos oficiais cumprem as condições naturais para que o conteúdo (informação) seja, primeiramente, sensível, percebida, classificada como positiva, focada e posteriormente, processada pelo cérebro, a partir da articulação entre as memórias (curto e longo prazo - conhecimentos prévios), funções executivas e aprendizagem.

Por esse motivo, justifica-se a inserção das contribuições da Neurociência Cognitiva, por ser inédita tal articulação e, também, por ser o cérebro o órgão mais importante da cognição, justificando as raízes da pretendida autonomia intelectual propostas nas diferentes tarefas no Ensino Médio e no Ensino Superior.

Diante desse preâmbulo e das teorias apanhadas para alicerçar a pesquisa em tela, deu-se continuidade a redação apresentando um breve resumo sobre as mesmas para que se tenha uma melhor compreensão dos argumentos que nelas repousam para analisar a transição do Ensino Médio para o Ensino Superior sempre que seja referido às Funções Trigonométricas.

\subsection{Teoria Antropológica do Didático: alguns elementos}

Ao ingressar na instituição "escola" o indivíduo (portador do meio interno, o cérebro) já encontra uma estrutura organizacional (meio externo, as instituições) pensada para auxiliar o seu desenvolvimento sócio-neurocognitivo por meio, inclusive, da AM.

Verificou-se que essa mesma estrutura repousa sobre os pressupostos epistemológicos, de forma que, para analisá-los recorreu-se aos fundamentos da Teoria Antropológica do Didático TAD, idealizada em 1998, pelo matemático francês Yves Chevallard. Até a presente data, não há registros de uma teoria desenvolvida para analisar a AM que se utilizasse da própria estrutura matemática que, segundo o autor, passou a observar, com as lentes de um matemático a Atividade Humana (AH), bem como as suas conformações, a exemplo das Instituições Sociais (IS), percebendo uma possibilidade de articulação entre esses três distintos e, ao mesmo tempo, análogos planos.

Chevallard [2] denominou de praxeologia um modelo teórico, desenvolvido para compreender qualquer atividade regular humana, postulando que a premissa básica da TAD pressupõe a existência de uma praxeologia ou organização praxeológica. No entendimento desse autor, a palavra praxeologia decorre do grego, como sendo: práxis (ação, hábito ou prática) + logos (conhecimentos, ciências ou teorias).

Desse modo, uma ação (práxis) é constituída por meio das noções de tarefas (t) e tipos de tarefas (T), cujo sentido e significado do conhecimento (logos) utilizado para o desenvolvimento 
de uma tarefa t é reconhecido pela existência de um discurso matemático alicerçado e disponível no interior do conhecimento empregado que justifica e interpreta a ação. Esse discurso Chevallard [2] designou de técnica $(\tau)$ - do grego tekhnê, saber-fazer.

Dessa forma, Chevallard (1998) configura a primeira parte de uma praxeologia relativa ao tipo de tarefa $\mathrm{T}$ que contém uma técnica $(\tau)$ respectiva a $\mathrm{T}$ : o bloco prático-técnico $[\mathrm{T} / \tau]$, denominado pelo autor de saber-fazer.

No entanto, conforme o autor, existe um discurso racional (logo) sobre a técnica $(\tau)$ que justifica e garante, racionalmente, a realização de um tipo de tarefa $\mathrm{T}$ por muitos, permitindo que sejam capazes de desenvolver as tarefas do mesmo tipo, denominado por ele de tecnologia $(\theta)$ que também deve tornar inteligível $\tau$ e explicar porque sua ocorrência.

Com efeito, esta dinâmica atmosfera denominada de $\theta$, sustenta-se sobre uma fonte/corpus/nível superior de conhecimento (justificativa-explicação-produção) e que ao mesmo tempo permite questionar o porquê desse discurso produzido nessa definição. Esse último nível corresponde ao discurso tecnológico ou à tecnologia da tecnologia, denominada por Chevallard [2] de teoria, representada por $\Theta$. Salienta-se, então, que a constituição dessa segunda parte de uma praxeologia de uma tarefa especifica dada, foi denominada pelo autor de bloco tecnológicoteórico $[\theta / \Theta]$, sinônimo de saber. Reunindo todos esses elementos, tem-se constituído uma praxeologia pontual representada por $[\mathrm{T} / \tau / \theta / \Theta]$, ou seja, uma praxeologia relativa a um único tipo de tarefa T.

Mas, antes mesmo desse pesquisador desenvolver a TAD, revelou em 1992 noções contidas em teorias da Antropologia Cognitiva que, segundo ele, referem-se a objetos, pessoas, instituições, relações que embasaram as primeiras formulações da Teoria da Transposição Didática - TTD, cujo intento foi compreender e unificar as diversas relações entre os fenômenos didáticos.

Para Chevallard [17], os objetos, seguramente, constituem em si mesmo, material de base de toda construção teórica. Desta forma, tudo é objeto: as pessoas X, as instituições I, são, por exemplo, de um tipo particular. E, por esse axioma, postula que um objeto só existirá desde que uma pessoa $X$ ou uma instituição I reconheça tal objeto como existente. Para tanto, é necessário e suficiente existir pelo menos uma relação de $\mathrm{O}$ com $\mathrm{X}, \mathrm{R}(\mathrm{X}, \mathrm{O})$, ou de $\mathrm{O}$ com I, RI(O), para que $\mathrm{O}$ exista. Foram essas noções que permitiram o mapeamento analítico das praxeologias existentes na Atividade Matemática, especificamente, no campo das Funções Trigonométricas.

$\mathrm{O}$ recuo a tais noções permitiu que o autor questionasse de que são constituídas e como seria possível projetar e executar uma técnica $(\tau)$. Para tanto, recorre a sua concepção de objeto, mantendo a sua teoria fechada e protegida, estabelecendo uma diferença essencial entre dois tipos específicos de objetos (quanto a Natureza): os objetos ostensivos e os objetos não ostensivos que serão melhores compreendidos e definidos, a partir das reflexões de Chevallard [1] e Bosch e Chevallard [21] quando articulam essas noções, as noções de relação a um objeto e praxeologia, bem como o seu papel na TAD - salienta-se, neste momento, a primeira articulação entre elementos teóricos da Antropologia Cognitiva, TTD, TAD, Relações Pessoais e Institucionais, objetos ostensivos e não ostensivos que estão disponíveis em Chevallard $(1,17)$ e em Bosch e Chevallard [21].

Dessa forma, Chevallard [1] define objetos ostensivos aqueles que têm para nós uma forma sensível ou material. Por conta dessas propriedades, o pesquisador subclassificou-os de:

- ostensivos materiais: uma caneta, um compasso etc.;

- ostensivos gestuais: os gestos;

- ostensivos discursivos: as palavras, e, mais genericamente, o discurso;

- ostensivos gráficos: os esquemas, desenhos, grafismos;

- ostensivos escriturais: as escritas e os formalismos.

Convém frisar que, dadas essas características, das subclassificações associadas aos seus respectivos exemplos, quaisquer uns desses ostensivos podem ser manipulados. Designados aqui por: Oo $\rightarrow \mathrm{m}$, objeto ostensivo manipulável. Contrariamente, os objetos não ostensivos que, para o autor, usualmente designamos de noções, conceitos, ideias etc., são pouco prováveis de serem manipulados, mas tão somente evocados por meio da manipulação dos ostensivos possivelmente associados. Assim, representados por: Ono $\rightarrow$ e, objeto não ostensivo evocado. 


\subsection{Os níveis de funcionamento do conhecimento}

Sendo a sala de aula de Matemática o ambiente didático formado por recursos, professores e alunos, para tornar possível o encontro entre os meios interno (o cérebro, especificamente, dos alunos) e externo (as instituições, especificamente, os documentos institucionais: programas de ensino, livro didático e avaliações), verificou-se nas abordagens da pesquisadora francesa Aline Robert a possibilidade de investigar como as tarefas apresentadas aos estudantes se comportam em relação à estrutura hierárquica decorrente de uma análise epistemológica diretamente, vinculada às noções das Funções Trigonométricas. Salienta-se aqui a segunda articulação entre elementos teóricos da TAD, Relações Pessoais e Institucionais, objetos ostensivos e não ostensivos disponíveis em Chevallard [1, 2, 17] e em Bosch e Chevallard [21] e os NFC de Robert [3,4], relativos aos tipos de Tarefas dirigidas às noções de funções trigonométricas que solicitam a manipulação dos objetos ostensivos sob a evocação dos objetos não ostensivos.

Os NFC podem ser compreendidos como um conjunto de ferramentas desenvolvidas por Robert [3, 4] para constituir uma análise epistemológica e didática e, com isso, organizar a hierarquia dos conhecimentos matemáticos que serão apresentados aos alunos, tanto do Ensino Médio como os do Ensino Superior.

Essas ferramentas auxiliam a preparação dos cenários de aprendizagem, preocupação da pesquisadora, cuja hierarquização do conhecimento permitiu, como em uma engrenagem, compreender o desenvolvimento do mesmo a partir de três níveis, a saber: Nível Técnico (NT), Nível Mobilizável (NM) e Nível Disponível (ND). Segundo a autora, tais instrumentos objetivam identificar o nível operacional de funcionamento dos conhecimentos, nas tarefas propostas, seja nos livros didáticos de matemática, nas macro avaliações ou ainda, na formulação de tarefas que ajudem identificar em que etapa do desenvolvimento matemático se encontram os estudantes da disciplina Cálculo I no Ensino Superior - caso particular da parte experimental dessa pesquisa.

De acordo com Robert [3, 4], existe uma hierarquia natural entre esses níveis que, caso sejam respeitados, auxiliam os estudantes a compreensão das noções matemáticas em jogo.

O primeiro deles é o NT que, segundo Robert [3], corresponde à aplicação imediata de teoremas, propriedades, definições e fórmulas, na resolução de uma tarefa que pretende mobilizar a Atividade Matemática (AM) dos alunos. Cabe ainda salientar que, nesse nível, a AM representa um trabalho isolado, local e concreto. A Figura 1 apresenta um exemplo com a noção das Funções Trigonométricas nesse nível da AM:

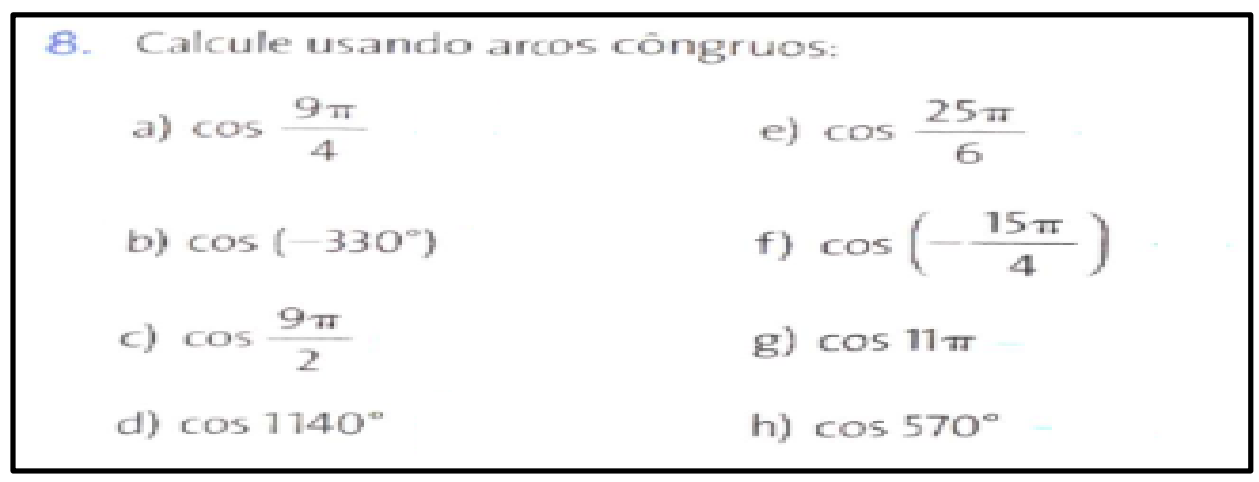

Figura 1: Exemplo do NT da Atividade Matemática relacionada às noções em jogo.

$\mathrm{O}$ segundo, NM que, de acordo com a autora, obedece a um funcionamento mais amplo que o NT por meio de aplicações simples, em que as propriedades sejam inseridas uma de cada vez, de forma que o questionável esteja explicitamente, descrito. Analogamente ao procedimento do nível anterior, o exemplo da Figura 2 mostrará como a noção matemática em tela, pode ser identificada nesse nível da AM: 


\section{Determine os valores reais de $m$ para que exista um número real $x$ que satisfaça as seguintes igualdades: \\ $\begin{array}{ll}\text { a) } \cos x=2 m+5 & \text { c) } \cos x=1-m^{2}\end{array}$ \\ b) $\cos x=3 m+4$ \\ d) $\cos x+5 m=6$}

Figura 2: Exemplo do NM da Atividade Matemática relacionada às noções em jogo.

O terceiro, denominado pela pesquisadora de ND, o aluno deve ser capaz de resolver o que lhe é oferecido sem nenhuma indicação, descobrindo por si mesmo o conhecimento necessário para resolução da tarefa. Desse modo, este nível inclui a escolha de teoremas, técnicas e estratégias. Nesse caso, esse nível da AM pode ser exemplificado, conforme propõe a tarefa apresentada na Figura 3:

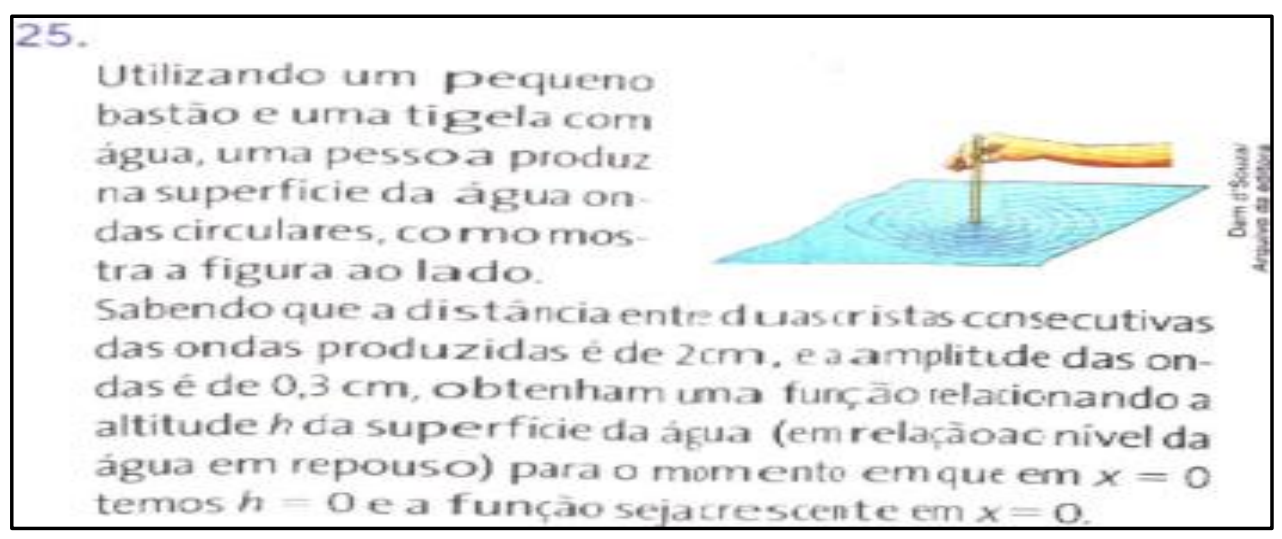

Figura 3: Exemplo do ND da Atividade Matemática relacionada às noções em jogo.

Neste cenário, foram identificadas algumas evidências nas abordagens de Robert [3, 4] e nas noções de Chevallard [1,2] e Bosch e Chevallard [21] que permitiram a entrada das perspectivas teóricas da Neurociência Cognitiva, quando consideram necessária a existência orquestrada de uma arquitetura neurocognitiva - responsável pelo processamento cerebral da informação - para tornar possível a realização de tarefas, sobretudo, aquelas provavelmente estruturadas por meio de uma organização praxeológica em torno de um tipo relativo às noções de funções trigonométricas que, teoricamente, cumpririam o seu papel institucional. Aqui, salienta-se a terceira articulação por meio de evidências encontradas nas definições ou propriedades nas abordagens dos NFC (técnico, mobilizável e disponível) de Robert [3,4] e nas noções de objetos ostensivos (manipuláveis) e não ostensivos (evocáveis) de Chevallard [1,2] e Bosch e Chevallard [21]. Dentre essas evidências, observou-se a necessidade de existência de episódios específicos na Memória de Longo Prazo (MLP), lócus natural para evocação dos objetos não ostensivos que se disponibilizados para as Funções Executivas, pincipalmente, pela via da flexibilidade cognitiva permite identificar o NFC e dispor os objetos ostensivos capazes de realizar um tipo de Tarefa relativa às noções de funções trigonométricas, por exemplo [6].

\subsection{Estrutura do funcionamento neurocognitivo}

É comum ouvir, em qualquer ocasião que requisite um indivíduo aprender sobre um determinado tema, a solicitação ou orientação: "preste ATENÇÃO”! Paralelamente e, geralmente, o "instrutor", "facilitador" ou "professor", também faz considerações sobre experiências anteriores do indivíduo que, em outras palavras, podem ser compreendidas como conhecimentos prévios (factual e procedimental) "armazenados" na Memória de Longo Prazo (MLP), caso existam. 
A esse respeito, o modelo de Willingham [23], representa adequadamente os pressupostos teóricos de Kandel [5] para compreender a estrutura do funcionamento neurocognitivo (Figura 4):

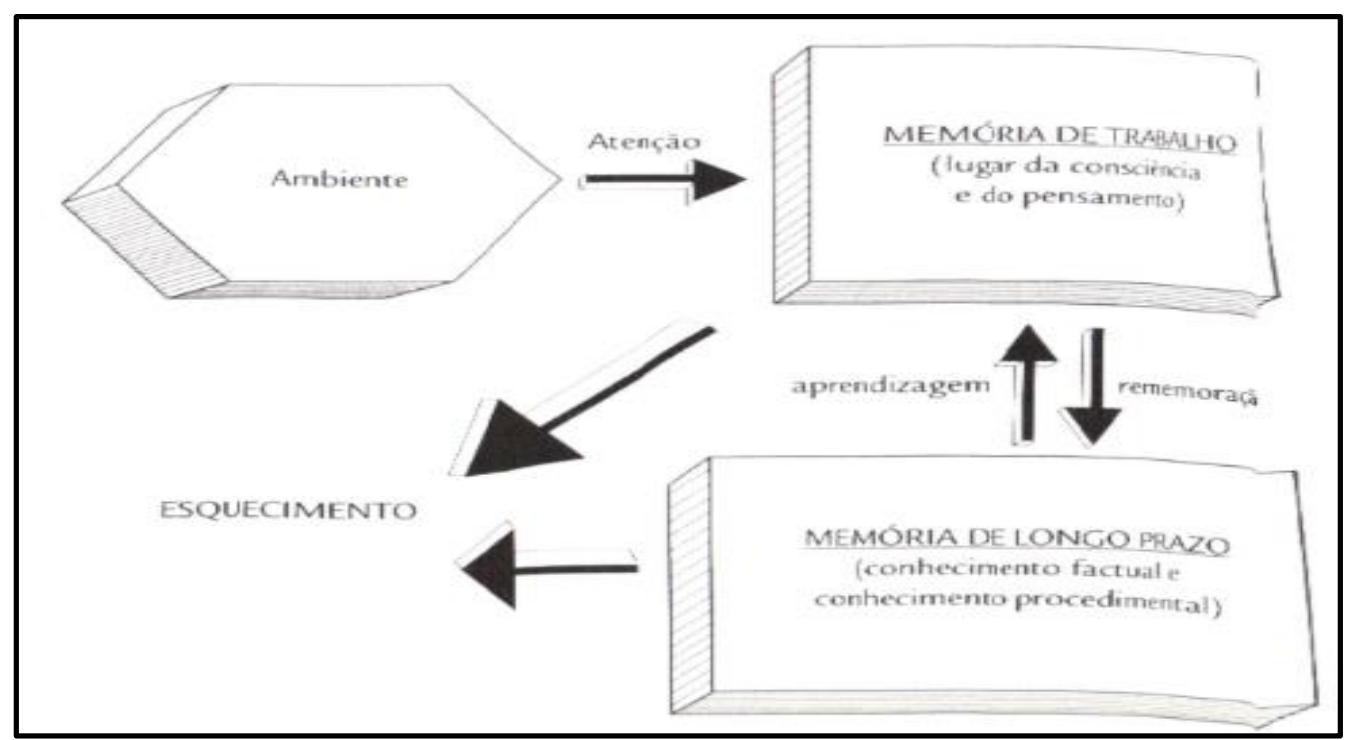

Figura 4 - Modelo do fluxo e da arquitetura cerebral da aquisição da informação.

Com efeito, residem em Luria [24], a partir da concepção de seus sistemas funcionais, as primeiras referências às funções cognitivas participantes da formação do pensamento e do processo de aprendizagem - atenção, percepção e memória, avaliadas pela bateria de testes (tarefas) neuropsicológicos produzidos pelo próprio autor. Nesse sentido, a pesquisadora Davidoff [25], denominou-as de "bases neuropsicológicas da aprendizagem".

Entretanto, a contínua investigação científica sobre essas primeiras funções cognitivas, detectou e integrou às mesmas, conforme resultados das investigações em Kandel [5], LeDoux [26], Gazzaniga [22], Lent [27, 28], Malloy-Diniz et al [29] e Willingham [23], um rol de outras funções cognitivas, passíveis de serem analisadas e avaliadas por meio de baterias de testes neuropsicológicos.

Um panorama atualizado de tais funções pode ser encontrado no trabalho de Fonseca [30, 31] que levou em consideração os testes neuropsicológicos disponíveis, selecionando-os e estabelecendo as articulações entre as funções cognitivas e as habilidades matemáticas, relacionadas às áreas de aritmética, álgebra e geometria.

A contínua investigação de Willingham [23], mesmo tendo referenciado as funções cognitivas atenção - tanto os estudos de Knudsen [32] quantos os de Lezak et al [33], reforçam o modelo de Willingham [23], pois demonstraram que a atenção como uma função cognitiva possibilita o acesso da informação "externa" à Memória de Curto Prazo/MCP (ou Memória Operacional), gerando respostas rápidas e adequadas - e MLP, importantes para a ocorrência da aprendizagem, ratifica os estudos de LeDoux [26] sobre a importância da emoção nesse processo, destacando em sua obra que "lembramos de coisas que causam alguma reação emocional." [23]. Diante desses achados, já se torna possível fazer um esboço parcial do fluxo das funções cognitivas ou circuitaria das funções cognitivas (Figura 5): 


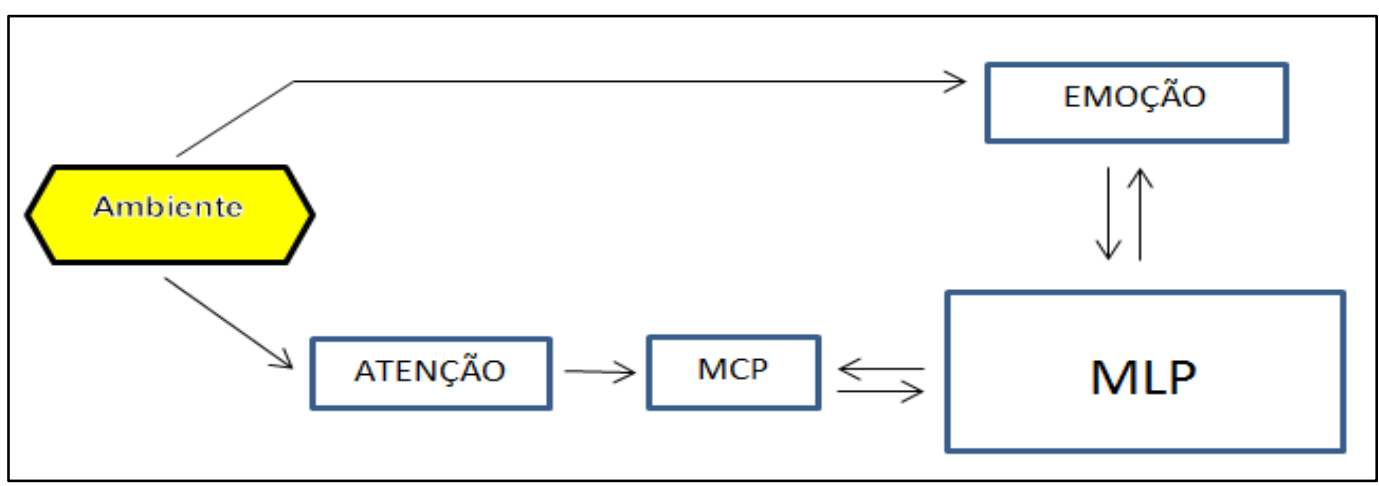

Figura 5 - Esboço parcial do fluxo das Funções Cognitivas.

Ainda assim, para que essa circuitaria possa ser concluída faz-se necessário observar que o contato inicial com o meio externo se dá pelas vias ou órgãos sensoriais, componentes do sistema somestésico: visão, audição, olfato, paladar e tato. Nesse momento, conforme postula LeDoux [26], a estimulação de um ou mais desses órgãos do sentido mobiliza outra função cognitiva - a percepção, responsável pela decodificação e interpretação dos fatos que, amalgamada à emoção, responsável pela classificação do sentido (sentimento positivo, neutro ou negativo), enviam uma mensagem para que o sistema atencional seja ativado.

Contempladas as duas primeiras unidades funcionais de Luria [24], precisa o cérebro, emitir os resultados dos seus pensamentos, transformando-os em comportamentos. Para dar cabo dessa tarefa, encontra-se no lobo frontal, área da planificação, capacidades cognitivas denominadas de funções executivas que responderam pela penúltima função cognitiva. Lent [28] as define como sendo "o conjunto de operações mentais que organizam e direcionam os diversos domínios cognitivos categoriais para que funcionem de maneira biologicamente adaptativa".

Contudo, observou-se na literatura que não existe um consenso entre os pesquisadores relativo aos componentes desse conjunto. Porém, conforme defende Malloy-Diniz et al. [29], é coerente preservar a lista que circula nas referências dos especialistas do assunto, como Lezak et al [33], Gazzaniga [22], Lent [28], Malloy-Diniz et al [29] e Miotto [34]. Nesse sentido, pode-se decompor as funções executivas em: planejamento, controle inibitório, tomada de decisões (julgamento), flexibilidade cognitiva, memória operacional, categorização e fluência.

Por fim, em Kandel [5] e, mais recentemente em Izquierdo [35] e Willingham [23], entendeuse à atribuição da aprendizagem (Ap) como o alicerce de todas as funções cognitivas. Nesses termos, salienta Willingham [23] que "o conteúdo a ser aprendido (isto é, a ser armazenado na memória de longo prazo), precisa passar algum período na memória de trabalho - ou seja, o aluno precisa prestar atenção". Dessa forma, o incremento desse recorte consente redimensionar a circuitaria das funções cognitivas conforme está apresentado na Figura 6:

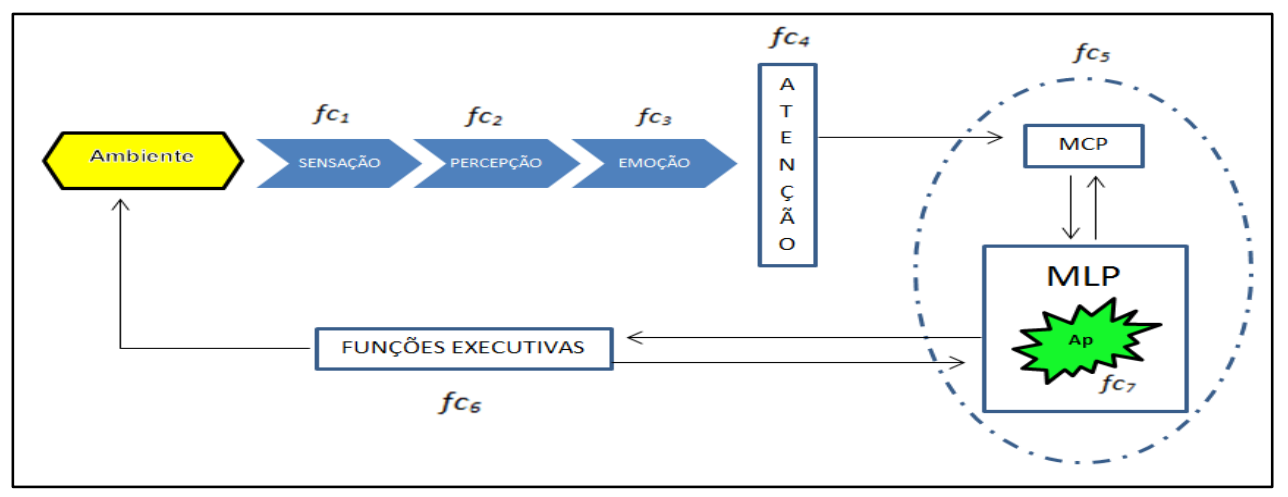

Figura 6 - Circuitaria das Funções Cognitivas.

Entendendo a circuitaria da Figura 6 como modelo contemporâneo de aquisição da informação, num nível mais profundo, seria necessário introduzir as noções de neuroquímicas para mostrar como essas funções se interligam e, dessa forma, como a informação é manipulada pelo cérebro. 
Isso permitirá as instituições escolares viabilizar e ressignificar alternativas pedagógicas, para que a informação intencional (as tarefas de Chevallard, por exemplo) represente para o cérebro, analogamente falando, "um prato de comida para quem tem fome".

A Figura 6 auxilia, por meio do binômio sensorial-motor, historicamente conhecido como estímulo-resposta, o alicerce da neuroquímica. Como células nervosas - que funcionam a base de estimulação - os neurônios (células especiais), descobertos por Waldeyer em 1891, juntamente com as células glias, compõem o tecido nervoso. Segundo Lent [27].

O neurônio é a principal unidade sinalizadora do sistema nervoso e exerce as suas funções com a participação dos gliócitos. É uma célula cuja morfologia está adaptada para as funções de transmissão e processamento de sinais: tem muitos prolongamentos próximos ao corpo celular (os dendritos), que funcionam como antenas para os sinais de outros neurônios, e um prolongamento longo que leva as mensagens do neurônio para sítios distantes (o axônio).

Os sinais captados no ambiente externo (as tarefas) são transduzidos para uma linguagem do cérebro através de substâncias químicas denominadas de neurotransmissores que cumprem seus papéis em áreas específicas do encéfalo. Neste sentido, o hipocampo é parte de uma estrutura do sistema límbico que está envolvido com os fenômenos da Memória de Longo Prazo.

\section{MATERIAL E MÉTODOS}

Considerando as reflexões acima, foi preciso que a investigação seguisse a metodologia: breve apresentação dos quadros teóricos escolhidos: meio externo (institucional) - Teoria Antropológica do Didático (TAD) de Yves Chevallard, abordagem dos Níveis de Funcionamento do Conhecimento (NFC) de Aline Robert que serviram como articuladores para compreender o meio interno (cérebro) - perspectivas teóricas da Neurociência Cognitiva, sob as lentes de Eric Kandel [5], principalmente; análise epistemológica das noções em jogo; as análises institucionais no Brasil e na França; análise neurocognitiva.

Para tanto, o material utilizado da primeira parte da pesquisa constituiu-se de documentos oficiais de ambos países (programas de ensino, livros didáticos e macro avaliações), tanto na Educação Básica como no Ensino Superior. Na segunda parte foram concebidos protocolos experimentais constituídos de três NFC (técnico, mobilizável, disponível) e aplicados em estudantes brasileiros e franceses do Ensino Superior para avaliar a possibilidade de existência de MLP nos extratos das amostras observadas.

\section{RESULTADOS E DISCUSSÃO}

Nesta seção, objetivou-se apresentar os resultados dos diferentes tipos de análise descritos acima.

A análise epistemológica foi realizada por meio de fontes bibliográficas que abordavam a história das noções de funções trigonométricas. As evidências identificadas apontaram para quatro transições epistemológicas dessas noções, conforme a Figura 7:

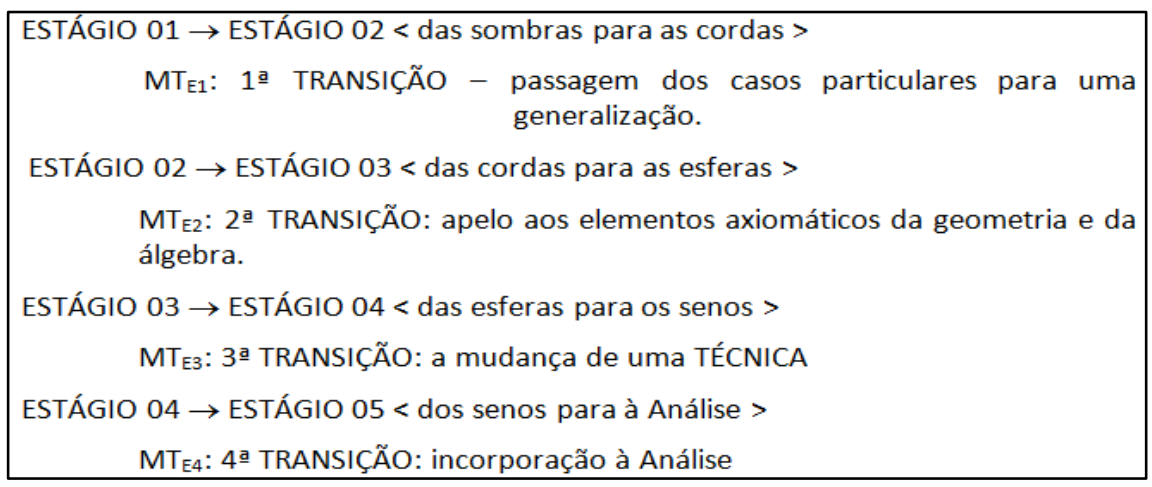

Figura 7 -Marcadores das Transições Epistemológicas $\left(M T_{E}\right)$. 
O principal fato encontrado foi a mudança de técnica $(\tau)$ empregada entre os ESTÁGIOS 02 e 03, conforme revela a Figura 7. Por sua vez, essa transformação justificou e assinalou uma ruptura entre as tecnologias $(\theta)$ disponíveis no ESTÁGIO 03 e as utilizadas no ESTÁGIO 04. Tal resultado fundamenta a resposta para a primeira questão de pesquisa.

Na sequência, foram desenvolvidas duas análises institucionais, Brasil e França, aplicando-se os princípios de uma organização praxeológica nos documentos oficiais de ambos países (programas de ensino, livros didáticos e macro avaliações), tanto na Educação Básica como no Ensino Superior.

Os resultados sintetizados revelaram que, no Brasil, é acentuada e potencializada a ruptura epistemológica em todos os documentos oficiais (programas de ensino, livros didáticos), pois a mudança entre os domínios da geometria plana para a função não se utiliza de tecnologias $(\theta)$ disponíveis em seus fundamentos teóricos/teoria $(\Theta)$, respectivamente, geometria e álgebra.

Quanto às macroavaliações, verificou-se que não refletem as expectativas esperadas para o Ensino Médio e, dessa forma, autentica, mesmo sem a validação desse conhecimento, o ingresso dos estudantes para o Ensino Superior. Com isso, o ENEM - Exame Nacional do Ensino Médio, não funciona como um filtro de estudantes, em função das expectativas institucionais, estabelecidas pelos programas oficiais de ensino e Livros Didáticos, nem como uma ferramenta de controle de qualidade desses mesmos agentes institucionais.

Nem no Ensino Superior, nem em macroavaliações de matemática ENADE - Exame Nacional de Desempenho de Estudantes, foi observado a existência de tipos de Tarefas que permitiriam o desenvolvimento de uma análise praxeológica em torno das noções das Funções Trigonométricas.

Mesmo assim, observou-se por meio dos levantamentos que a comparação entre o Ensino Médio e Ensino Superior só foi permitida a partir dos Programas de Ensino e dos Livros Didáticos. Dessa forma, tal comparação foi desenvolvida através do cruzamento - vale ressaltar que, conforme definição de Chevallard e Grenier [36], é a partir do cruzamento dos dados que se torna possível alcançar os objetivos de uma pesquisa documental - dos dados obtidos, referentes aos programas de ensino brasileiro, indicando-se a prevalência da mudança entre os domínios matemáticos geometria e função para abordar as noções trigonométricas iniciais, seno e cosseno, quando eram articuladas as noções de ângulos e a noção de número real, respectivamente.

$\mathrm{Na}$ França, sob os aspectos referentes aos documentos oficiais (programas de ensino, livros didáticos) do ensino secundário, verificou-se uma identidade com os resultados brasileiros: a ruptura entre os domínios matemáticos geometria e função para tratar das noções em jogo, quando eram articuladas as noções de ângulos e a noção de número real, respectivamente, também prevaleceram.

Quanto ao sistema de macroavaliações para a entrada na universidade, o BAC BACCALAURÉAT, equivalente, no Brasil, ao atual ENEM, constatou-se a ausência de tipos de Tarefas, que estivessem relacionadas às noções das Funções Trigonométricas. Analogamente, tais resultados se aplicaram aos programas de ensino, livro didático e macroavaliações do sistema de Ensino Superior francês.

Em um balanço entre os dois níveis de ensino, as evidências anteriores permitiram demonstrar que nos manuais do Collège (nível de ensino que equivale, no Brasil, ao Ensino Fundamental II), ratificaram que a mudança entre os domínios da geometria e da função, não é mobilizada por meio de organizações matemáticas que busquem ajustar a ruptura epistemológica identificada no estudo das noções das Funções Trigonométricas. Similarmente, nos manuais do Lycée (nível de ensino que equivale, no Brasil, ao Ensino Médio), existem sinais que corroboram a continuidade da ruptura identificada, cujo prejuízo é silenciosamente conduzido para o Ensino Superior.

Por sua vez, o Ensino Superior não considera importantes os conhecimentos relativos às noções das Funções Trigonométricas, pois parte do princípio estarem disponíveis para implementar, o aprofundamento das mesmas por meio das articulações entre geometria vetorial e números complexos, para a abordagem das noções relativas ao cálculo diferencial e integral, sobretudo, quando determinados tipos de Tarefas exigem a aplicação dessas articulações, para a compreensão da fórmula de Euler que definiu as Funções Trigonométricas como sendo

$$
\operatorname{sen} \mathrm{z}=\frac{\mathrm{e}^{\mathrm{iz}}-\mathrm{e}^{-\mathrm{iz}}}{2 \mathrm{i}}, \cos \mathrm{z}=\frac{\mathrm{e}^{\mathrm{iz}}+\mathrm{e}^{-\mathrm{iz}}}{2} \text { e } \mathrm{e}^{\mathrm{z}}=\lim _{\mathrm{n} \rightarrow \infty}\left(1+\frac{\mathrm{z}}{\mathrm{n}}\right)^{\mathrm{n}}, \text { conforme Kennedy [7]. }
$$


Sob o abrigo dessas evidências, tanto o sistema brasileiro quanto o francês, não conseguem neutralizar a ruptura epistemológica - mudança de técnica $(\tau)$ empregada entre os ESTÁGIOS 02 e 03 -, cujas tecnologias $(\theta)$ disponíveis nos referidos estágios não dialogavam entre si. Tal resultado alicerça, do ponto de vista institucional, a resposta para a primeira questão de pesquisa.

Por fim, a análise neurocognitiva buscou considerar os princípios do funcionamento neurocognitivo, especificamente, em uma de suas funções - a Memória de Longo Prazo - para verificar a possibilidade de auxiliar no processo de neutralização da ruptura identificada em atividades matemáticas concebidas na articulação entre TAD e os NFC indicada no item 1.4 deste artigo.

Os resultados decorrentes dos diagnósticos de protocolos experimentais aplicados em estudantes brasileiros e franceses do Ensino Superior revelaram que em tarefas elaboradas segundo os três NFC (técnico, mobilizável, disponível), foi mínima a existência de MLP nos extratos das amostras observadas, justificadas pelas declarações dos estudantes voluntários por não "lembrarem" das referidas noções ou pela ausência de manipulação de ostensivos adequados na maior parte dos extratos dessas amostras.

Sendo assim, foi possível constatar que, tanto o Ensino Médio quanto o Ensino Superior não levam em consideração os princípios da Neurociência Cognitiva que, por meio da existência solidificada da MLP, auxiliaria com mais eficácia o processo de neutralização da ruptura epistemológica, na transição do ensino das Funções Trigonométricas do EM-ES.

Esse fato consentiu concluir que se faz necessário institucionalizar, desde o início do processo de aprendizagem, os princípios da Neurociência Cognitiva, cujas possibilidades de sucesso podem ajudar a modificar, os casos onde a aprendizagem não foi verificada. Com efeito, respondeu-se, dessa forma a segunda questão de pesquisa.

\section{CONCLUSÃO}

A pesquisa apresentada neste artigo procurou analisar a Transição do Ensino das Funções Trigonométricas EM-ES sob a ótica da articulação entre os quadros da Didática da Matemática e da Neurociência Cognitiva.

Seguindo uma metodologia direcionada pelos quadros teóricos relacionados no texto e aplicada nos níveis de Ensino Médio e Superior por meio de documentos oficiais do ensino brasileiro e francês, em livros didáticos de matemática de ambas nacionalidades e do processamento cerebral da Atividade Matemática por meio da Memória de Longo Prazo (MLP) em estudantes do Brasil e da França, concluiu-se que as causas da ruptura existente no processo de transição EM-ES relativa as noções de funções trigonométricas deve-se a mudança de técnicas $(\tau)$ empregadas entre a evolução dos estágios epistemológicos dessas noções, principalmente, por não serem ativadas sob um mesmo discurso tecnológico $(\theta)$.

Como esse tipo de ruptura - epistemológica -, que foi até o momento, silenciosamente preservada e sobrevive nos sistemas de ensino dos países analisados, contribui para que a transição ocorra de maneira lenta (no Brasil) e média (na França), respectivamente, considerando, teoricamente, os documentos oficiais; na mesma sequência, de modo médio e alto, analisando-se os livros didáticos e, por fim, em ritmo médio nos dois territórios, quando enfocou-se nos ostensivos e não ostensivos vinculados as noções de funções trigonométricas existentes e disponíveis na MLP dos estudantes investigados.

Esse conjunto de fatos permitiu concluir que a transição do ensino das noções de funções trigonométricas apesar de acontecer nos países analisados de modos diferentes, preserva uma causa singular. Além disso, não consideram institucionalizados os princípios da Neurociência Cognitiva como uma possibilidade para ajudar a neutralizar o tipo de ruptura existente.

\section{REFERÊNCIAS BIBLIOGRÁFICAS}

1. Chevallard, Y. Ostensifs et non-ostensifs dans l'activité mathématique, Séminaire de l'Associazione Mathesis, Turin, 3 février 1994, in Actes du Séminaire 1993-1994, 190-200. 1994. 
2. Chevallard, Y. Analyse des pratiques enseignantes et didactique des mathématiques: l'approche anthropologique. Cours donné à l'université d'été Analyse des pratiques enseignantes et didactique des mathématiques, La Rochelle, 4-11 juillet 1998 ; paru dans les actes de cette université d'été, IREM de Clermont-Ferrand, p. 91-120.1998.

3. Robert, A. Quelques outils d'analyse épistemologique et didactique de connaissances mathématiques à enseigner au lycée et à l'université. Actes de la IX école d'ete de didactique dês mathématiques. França: Houlgate, 1997.

4. Robert, A. Outils d'analyse des contenus mathématiques à enseigner au lycée et à l'université. Recherches en Didactique des Mathématiques, [S.1.], v. 18, n. 2, p.139-190, 1998.

5. Kandel, E. et al. Principles of Neural Science. Nova York: McGraw-Hill, 1991.

6. Fonseca, L. S. da. Um estudo sobre o Ensino de Funções Trigonométricas no Ensino Médio e no Ensino Superior no Brasil e França. 2015, 1v. 495p. Tese de Doutorado. Universidade Anhanguera de São Paulo, São Paulo (SP).

7. Kennedy, ES. História da trigonometria. São Paulo: Atual, 1992. (Coleção Tópicos de História da Matemática para sala de aula; v. 5)

8. Sampaio, JL, Calçada, CS. Física, volume único. São Paulo: Atual, 2008.

9. Abdounur, OJ. Matemática e música: pensamento análogo na construção de significados. São Paulo: Escrituras Editora, 1999.

10. BRASIL. Secretaria de Educação Média e Tecnológica. Parâmetros Curriculares Nacionais Ensino Médio: Ciências da Natureza, Matemática e suas Tecnologias. Brasília: MEC / SEMT, 2000.

11. Nasser, L, Sousa, GA, TORRACA, MA. Transição do ensino médio para o superior: como minimizar as dificuldades em cálculo? In: V Seminário Internacional de Pesquisa em Educação Matemática (em CD). Petrópolis, RJ, 2012.

12. Rezende, W. O ensino de Cálculo: dificuldades de natureza epistemológica. In: Machado, NJ. e Cunha, MO: Linguagem, Conhecimento, Ação - Ensaios de Epistemologia e Didática, p. 313-336. Escrituras Editora, São Paulo, 2003.

13. Palis, G. A transição do Ensino Médio para o Ensino Superior. Anais do X Encontro Nacional de Educação Matemática (em CD). Salvador, BA, 2010.

14. Sierpinska, A. On understanding the notion of function. In: DUBINSKY, E; HAREL, G (Ed.) The Concept of Function: aspects of epistemology and Pedagogy. MAA Notes, 1992, p.25-58.

15. Fonseca, LS. Aprendizagem em Trigonometria: o olhar da Educação Matemática. Dissertação de Mestrado - Programa de Pós-Graduação em Educação, Universidade Federal de Sergipe, São Cristóvão, 2002.

16. Fonseca, LS. Aprendizagem em Trigonometria: obstáculos, sentidos e mobilizações. São Cristóvão: UFS, 2011.

17. Chevallard, Y. Concepts fondamentaux de la didactique: perspectives apportées par une approche anthropologique . Recherches en didactique des mathématiques. Grenoble: La Pensée Sauvage, 1992.

18. Chevallard, Y. Organiser l'étude: 3. Ecologie \& regulation: Actes XIe école d'été de didactique des mathématiques. Grenoble: La Pensée Sauvage, 2002a.

19. Chevallard, Y. Les mathématiques dans les formations universitaires: un schéma alternative. Notes pour exposé présenté au séminaire. Mathématiques et sciences humaines de la Faculté des sciences de Luminy, Méditerranée, 2007.

20. Chevallard, Y. Le développement actuel de la TAD: pistes et jalons. Notes pour un exposé donné le 6 juin 2007 au Séminaire DIDIREM (Université Paris 7), 2007a.

21. Bosch, M.; Chevallard, Y. La sensibilité de l'activité mathématique aux ostensifs. Objet d'étude et problématique. Recherches en Didactique des mathématiques, Paris, v. 19, n. 1, p. 77-123, 1999.

22. Gazzaniga, M. S. et al. Neurociência Cognitiva: a biologia da mente. Porto Alegre: Artmed, 2006.

23. Willingham, D. T. Por que os alunos não gostam da escola? Reposta da ciência cognitiva para tornar a sala de aula atrativa e efetiva. Porto Alegre: Artmed, 2011.

24. Luria, AR. Fundamentos de Neuropsicologia. São Paulo: USP, 1981.

25. Davidoff, LL. Introdução à Psicologia. São Paulo: Pearson Education Brasil, 2001.

26. LeDoux, J. O cérebro emocional: os misteriosos alicerces da vida emocional. Rio de Janeiro: Objetiva, 2001.

27. Lent, R. Cem bilhões de neurônios. Rio de Janeiro: Atheneu, 2002.

28. LEnt, R. Neurociência da Mente e do Comportamento. Rio de Janeiro: Guanabara Koogan, 2008.

29. Malloy-Diniz et al. Neuropsicologia das Funções Executivas. In: FUENTES, D. et al. (2008). Neuropsicologia: teoria e prática. Por Alegre: Artmed, 2008.

30. Fonseca, LS. Protocolo Neuropsicopedagógico de Avaliação Cognitiva das Habilidades Matemáticas. Rio de janeiro: WAK, 2013a. 
31. Fonseca, LS. A neuromatemática como um novo paradigma para a Educação Matemática. In: Direcional Educador (ISSN 1982-2898). São Paulo: Grupo Direcional, 2013b.

32. Knudsen, EI. Fundamental components of attention. Annual Review of Neuroscience, 2007, v. 30, p. 57-58.

33. Lezak, MD. et al. Neuropsychological assessment. New York: Oxford, 1976.

34. Miotto, EC. Avaliação neuropsicológica e Funções cognitivas. In: Miotto, EC, Lucia, M C, Scafe, M (org.). Neuropsicologia Clínica. São Paulo: Roca, 2012.

35. Izquierdo, I. Memória. Porto Alegre: Artmed, 2011.

36. Chevallard, Y; Grenier, D. Le topos de l' éleve, Actes de la IX école d' été de Didactique des Mathématiques, Houlgate, 1997. 